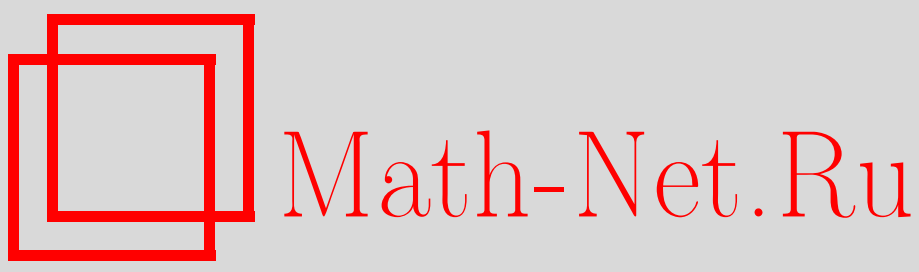

V. D. Stepanov, G. È. Shambilova, Multidimensional bilinear hardy inequalities, Sibirsk. Mat. Zh., 2020, Volume 61, Number 4, 913-931

DOI: https://doi.org/10.33048/smzh.2020.61.413

Use of the all-Russian mathematical portal Math-Net.Ru implies that you have read and agreed to these terms of use http://www . mathnet.ru/eng/agreement

Download details:

IP: 54.162 .85 .209

April 26, 2023, 13:26:50

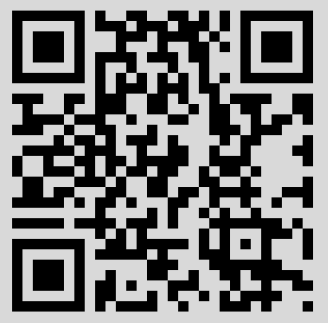


Сибирский математический журнал Июль-август, 2020. Том 61, № 4

УДК 517.51

\title{
МНОГОМЕРНЫЕ БИЛИНЕЙНЫЕ НЕРАВЕНСТВА ХАРДИ
}

\section{В. Д. Степанов, Г. Э. Шамбилова}

\begin{abstract}
Аннотация. Получена характеризация многомерного билинейного неравенства Харди в весовых пространствах Лебега.

DOI 10.33048/smzh.2020.61.413

Ключевые слова: весовое пространство Лебега, неравенства Харди, билинейный оператор.
\end{abstract}

\section{$\S 1$. Введение}

Пусть $\mathfrak{M}$ - множество всех измеримых по Лебегу функций на $\mathbb{R}^{n}, \mathfrak{M}^{+} \subset$ $\mathfrak{M}$ - подмножество всех неотрицательных функций. При $v \in \mathfrak{M}^{+}$и $0<p \leq \infty$ определим весовое пространство Лебега

$$
\begin{gathered}
L_{v}^{p}:=\left\{f \in \mathfrak{M}:\|f\|_{p, v}:=\left(\int_{\mathbb{R}^{n}}|f(x)|^{p} v(x) d x\right)^{\frac{1}{p}}<\infty\right\}, \quad 0<p<\infty, \\
L_{v}^{\infty}:=\left\{f \in \mathfrak{M}:\|f\|_{\infty, v}:=\underset{x \in \mathbb{R}^{n}}{\operatorname{ess} \sup } v(x)|f(x)|<\infty\right\}, \quad p=\infty .
\end{gathered}
$$

Многомерным оператором Харди называется преобразование

$$
H f(x)=\int_{B(|x|)} f(y) d y,
$$

где $B(|x|):=\left\{y \in \mathbb{R}^{n}:|y|<|x|\right\}$. Исследованию ограниченности оператора (1) в весовых пространствах Лебега, а также в других функциональных классах посвящено большое количество работ (см., например, [1-7]).

Для произвольных фиксированных параметров $0<q \leq \infty, 1 \leq p_{1}, p_{2} \leq \infty$ и весовых функций $v_{1}, v_{2}, u \in \mathfrak{M}^{+}$рассмотрим задачу характеризации билинейного неравенства

$$
\|H f \cdot H g\|_{q, u} \leq C\|f\|_{p_{1}, v_{1}}\|g\|_{p_{2}, v_{2}}, \quad f, g \in \mathfrak{M}^{+},
$$

где константа $C$ не зависит от функций $f, g$ и полагается наименьшей из возможных.

Работа В. Д. Степанова (теоремы 2, 3, 13, и 14) финансировалась из гранта Российского научного фонда (проект 19-11-00087) и выполнялась в Математическом институте им. В. А. Стеклова РАН. По остальной части статьи работа В. Д. Степанова выполнялась в рамках государственного задания Министерства науки и высшего образования РФ для Вычислительного центра ДВО РАН. Результаты работы Г. Э. Шамбиловой частично поддержаны грантом РФФИ (проект 19-01-00223).

(C) 2020 Степанов В. Д., Шамбилова Г. Э. 
В одномерном случае аналогичная задача с операторами интегрирования $\mathscr{H} f(x):=\int_{0}^{x} f$ изучалась в $[8,9]$ как дополнение к некоторым результатам о мультилинейных неравенствах [10,11]. Для операторов Харди - Стеклова [12] этот случай рассмотрен в $[13,14]$. Билинейные весовые неравенства с операторами Вольтерра

$$
R_{i} f(x):=\int_{0}^{x} k_{i}(x, y) f(y) d y, \quad R_{i}^{*} f(x):=\int_{x}^{\infty} k_{i}(y, x) f(y) d y, \quad i=1,2,
$$

изучены в [15-18], а аналогичная задача на конусе монотонных функций - в [19-21].

Неравенство (2) для некоторых значений параметров суммирования независимо изучалось в $[22,23]$. В [22] для доказательств использовался метод дискретизации-антидискретизации, заложенный в [24,25], а в [23] применялась редукция к одномерному неравенству.

В данной работе используется прямой метод характеризации билинейного неравенства (2), основанный на применении критериев ограниченности многомерного оператора Харди. Представлены критерии для всех возможных значений параметров суммирования, включая новый случай $0<q<1, p_{i}=1$, $i=1,2$.

Результаты данной статьи анонсированы в [26].

Для характеризации билинейных неравенств (2) применяем критерии выполнения многомерного весового неравенства Харди [2], полученные при $1<p \leq$ $q<\infty$ и $1<q<p<\infty$. Дополнительно к этому в данной работе по аналогии с [27] рассмотрены случаи $0<q<p, 1<p<\infty$ и $0<q<1, p=1$. Для крайних значений параметров используются классические результаты [28, гл. 11] (см. также [29, теорема 1.1]).

Основные результаты содержатся в $\S 3$.

Всюду в работе произведения вида $0 \cdot \infty$ полагаются равными 0 и $g^{\alpha}(x):=$ $[g(x)]^{\alpha}$. Соотношение $A \lesssim B$ означает $A \leq c B$ с константой $c$, зависящей только от параметров суммирования; $A \approx B$ равносильно $A=c B$ или $A \lesssim B \lesssim A$. Если $1<p<\infty$, то $p^{\prime}:=\frac{p}{p-1}$.

\section{$\S$ 2. Весовые неравенства Харди}

В этом параграфе будут дополнены результаты о неравенстве

$$
\left(\int_{\mathbb{R}^{n}}\left(\int_{B(|x|)} f\right)^{q} u(x) d x\right)^{\frac{1}{q}} \leq C\left(\int_{\mathbb{R}^{n}} f^{p} v\right)^{\frac{1}{p}}, \quad f \in \mathfrak{M}^{+},
$$

полученные в $[2, \S 2]$ для $1<p, q<\infty$, новыми случаями отношений параметров $p$ и $q$, исчерпывающими весь диапазон их возможных изменений $0<q \leq \infty$, $1 \leq p \leq \infty$. При $0<p<1$ неравенство (3) выполняется лишь в тривиальном случае.

Теорема 1. Пусть $0<q<p, 1<p<\infty$ и $\frac{1}{r}:=\frac{1}{q}-\frac{1}{p}$. Тогда для наилучшей константы $C$ в неравенстве (3) выполнено соотношение

$$
C \approx\left(\int_{\mathbb{R}^{n}}\left(\int_{B(|x|)} v^{1-p^{\prime}}\right)^{\frac{r}{p^{\prime}}}\left(\int_{\mathbb{R}^{n} \backslash B(|x|)} u\right)^{\frac{r}{p}} u(x) d x\right)^{\frac{1}{r}}=: D .
$$


ДокАЗАТЕЛЬСТво. ОцЕНКА СНИЗУ. Предположим, что неравенство (3) верно, и обозначим

$$
w:=v^{1-p^{\prime}}, \quad \mathbf{u}(t):=\int_{\Sigma_{n-1}} t^{n-1} u(t \tau) d \tau, \quad \mathbf{w}(t):=\int_{\Sigma_{n-1}} t^{n-1} w(t \tau) d \tau .
$$

Пусть

тогда

$$
f(y)=\left(\int_{\mathbb{R}^{n} \backslash B(|y|)} u\right)^{\frac{r}{p q}}\left(\int_{B(|y|)} w\right)^{\frac{r}{p q^{\prime}}} w(y),
$$

$$
\begin{gathered}
\int_{B(|x|)} f(y) d y=\int_{B(|x|)}\left(\int_{\mathbb{R}^{n} \backslash B(|y|)} u\right)^{\frac{r}{p q}}\left(\int_{B(|y|)} w\right)^{\frac{r}{p q^{\prime}}} w(y) d y \\
\geq\left(\int_{\mathbb{R}^{n} \backslash B(|x|)} u\right)^{\frac{r}{p q}} \int_{B(|x|)}\left(\int_{B(|y|)} w\right)^{\frac{r}{p q^{\prime}}} w(y) d y \\
=\left(\int_{\mathbb{R}^{n} \backslash B(|x|)} u\right)^{\frac{r}{p q}} \int_{0}^{|x|} \int_{\Sigma_{n-1}} t^{n-1} w(t \tau)\left(\int_{0}^{t} \int_{\Sigma_{n-1}} s^{n-1} w(s \sigma) d s d \sigma\right)^{\frac{r}{p q^{\prime}}} d t d \tau \\
=\left(\int_{\mathbb{R}^{n} \backslash B(|x|)} u\right)^{\frac{r}{p q}} \int_{0}^{|x|} \mathbf{w}(t)\left(\int_{0}^{t} \mathbf{w}(s) d s\right)^{\frac{r}{p q^{\prime}}} d t \\
=\frac{p^{\prime} q}{r}\left(\int_{\mathbb{R}^{n} \backslash B(|x|)} u\right)^{\frac{r}{p q}}\left(\int_{0}^{|x|} \mathbf{w}(t) d t\right)^{\frac{r}{p^{\prime} q}}=\frac{p^{\prime} q}{r}\left(\int_{\mathbb{R}^{n} \backslash B(|x|)} u\right)^{\frac{r}{p q}}\left(\int_{B(|x|)} w\right)^{\frac{r}{p^{\prime} q}} .
\end{gathered}
$$

Применяя полученную оценку в неравенстве (3), получим

$$
\begin{aligned}
D^{\frac{r p}{q}}=\left(\int_{\mathbb{R}^{n}}\left(\int_{\mathbb{R}^{n} \backslash B(|x|)} u\right)^{\frac{r}{p}}\left(\int_{B(|x|)} w\right)^{\frac{r}{p^{\prime}}} u(x) d x\right)^{\frac{p}{q}} \\
\quad \lesssim\left(\int_{\mathbb{R}^{n}}\left(\int_{B(|x|)} f\right)^{q} u(x) d x\right)^{\frac{p}{q}} \leq C^{p} \int_{\mathbb{R}^{n}} f^{p} v \\
=C^{p} \int_{\mathbb{R}^{n}}\left(\int_{\mathbb{R}^{n} \backslash B(|x|)} u\right)^{\frac{r}{q}}\left(\int_{B(|x|)} w\right)^{\frac{r}{q^{\prime}}} w(x) d x \\
=C^{p} \int_{0}^{\infty} \mathbf{w}(t)\left(\int_{t}^{\infty} \mathbf{u}\right)^{\frac{r}{q}}\left(\int_{0}^{t} \mathbf{w}\right)^{\frac{r}{q^{\prime}}} d t \\
\approx C^{p} \int_{0}^{\infty} \mathbf{u}(s)\left(\int_{s}^{\infty} \mathbf{u}\right)^{\frac{r}{p}} s\left(\int_{0}^{t} \mathbf{w}\right)^{\frac{r}{q^{\prime}}} \mathbf{w}(t) d t d s \\
\lesssim C^{p} \int_{0}^{\infty}\left(\int_{s}^{\infty} \mathbf{u}\right)^{\frac{r}{p}}\left(\int_{0}^{s} \mathbf{w}\right)^{\frac{r}{p^{\prime}}} \mathbf{u}(s) d s
\end{aligned}
$$




$$
=C^{p} \int_{\mathbb{R}^{n}}\left(\int_{\mathbb{R}^{n} \backslash B(|x|)} u\right)^{\frac{r}{p}}\left(\int_{B(|x|)} w\right)^{\frac{r}{p^{\prime}}} u(x) d x=C^{p} D^{r} .
$$

Разделив обе части неравенства на $D^{r}$, имеем $D \lesssim C$.

Оценка СВеРху. Пусть $D<\infty$, обозначим

$$
\mathbf{f}(s):=\int_{\Sigma_{n-1}} s^{n-1} f(s \sigma) d \sigma, \quad \mathbf{W}(s):=\int_{0}^{s} \mathbf{w}(t) d t
$$

и временно предположим, что

$$
\int_{0}^{\infty} \mathbf{w}(t) d t=\infty .
$$

Воспользуемся утверждениями 2.2, 2.3 из [27]. Тогда в левой части неравенства (3) имеем

$$
\begin{aligned}
& \left(\int_{\mathbb{R}^{n}}\left(\int_{B(|x|)} f\right)^{q} u(x) d x\right)^{\frac{1}{q}} \\
& =\left(\int_{0}^{\infty} \int_{\Sigma_{n-1}} t^{n-1} u(t \tau) d \tau\left(\int_{0}^{t} \int_{\Sigma_{n-1}} s^{n-1} f(s \sigma) d s d \sigma\right)^{q} d t\right)^{\frac{1}{q}} \\
& =\left(\int_{0}^{\infty} \mathbf{u}(t)\left(\int_{0}^{t} \mathbf{f}(s) d s\right)^{q} d t\right)^{\frac{1}{q}} \\
& \lesssim\left(\int_{0}^{\infty}\left(\int_{t}^{\infty} \mathbf{u}\right)^{\frac{r}{q}}\left(\int_{t}^{\infty} \mathbf{W}^{-p} \mathbf{W}\right)^{-\frac{r}{q}} \mathbf{W}^{-p}(t) \mathbf{w}(t) d t\right)^{\frac{1}{r}} \\
& \times\left(\int_{0}^{\infty}\left(\int_{0}^{t} \mathbf{f}\right)^{p} \mathbf{W}^{-p}(t) \mathbf{w}(t) d t\right)^{\frac{1}{p}} \\
& \lesssim\left(\int_{0}^{\infty}\left(\int_{t}^{\infty} \mathbf{u}\right)^{\frac{r}{q}}\left(\int_{0}^{t} \mathbf{w}\right)^{\frac{r}{q^{\prime}}} \mathbf{w}(t) d t\right)^{\frac{1}{r}}\left(\int_{0}^{\infty} \mathbf{f}^{p} \mathbf{w}^{1-p}\right)^{\frac{1}{p}} \\
& \approx\left(\int_{0}^{\infty}\left(\int_{t}^{\infty} \mathbf{u}\right)^{\frac{r}{p}}\left(\int_{0}^{t} \mathbf{w}\right)^{\frac{r}{p^{\prime}}} \mathbf{u}(t) d t\right)^{\frac{1}{r}}\left(\int_{0}^{\infty} \mathbf{f}^{p} \mathbf{w}^{1-p}\right)^{\frac{1}{p}} \\
& =\left(\int_{0}^{\infty} \int_{\Sigma_{n-1}} t^{n-1} u(t \tau) d \tau\left(\int_{t}^{\infty} \int_{\Sigma_{n-1}} t^{n-1} u(s \sigma) d s d \sigma\right)^{\frac{r}{p}}\right. \\
& \left.\times\left(\int_{0}^{t} \int_{\Sigma_{n-1}} t^{n-1} w(s \sigma) d s d \sigma\right)^{\frac{r}{p^{\prime}}} d t\right)^{\frac{1}{r}}
\end{aligned}
$$




$$
\begin{aligned}
& \times\left(\int_{0}^{\infty}\left(\int_{\Sigma_{n-1}} t^{n-1} f(t \tau) d \tau\right)^{p}\left(\int_{\Sigma_{n-1}} t^{n-1} w(t \tau) d \tau\right)^{1-p} d t\right)^{\frac{1}{p}} \\
& \quad \leq\left(\int_{\mathbb{R}^{n}}\left(\int_{B(|x|)} v^{1-p^{\prime}}\right)^{\frac{r}{p^{\prime}}}\left(\int_{\mathbb{R}^{n} \backslash B(|x|)} u\right)^{\frac{r}{p}} u(x) d x\right)^{\frac{1}{r}}\left(\int_{\mathbb{R}^{n}} f^{p} w^{1-p}\right)^{\frac{1}{p}} .
\end{aligned}
$$

В последнем неравенстве применяется неравенство Гёльдера

$$
\begin{aligned}
& \int_{0}^{\infty}\left(\int_{\Sigma_{n-1}} t^{n-1} f(t \tau) d \tau\right)^{p}\left(\int_{\Sigma_{n-1}} t^{n-1} w(t \sigma) d \sigma\right)^{1-p} d t \\
&=\int_{0}^{\infty} t^{n-1}\left(\int_{\Sigma_{n-1}} f(t \tau) w^{\frac{1-p}{p}}(t \tau) w^{-\frac{1-p}{p}}(t \tau) d \tau\right)^{p}\left(\int_{\Sigma_{n-1}} w(t \sigma) d \sigma\right)^{1-p} d t \\
& \leq \int_{0}^{\infty} t^{n-1}\left(\int_{\Sigma_{n-1}} f^{p}(t \tau) w^{1-p}(t \tau) d \tau\right) d t=\left(\int_{\mathbb{R}^{n}} f^{p} w^{1-p}\right)^{\frac{1}{p}} .
\end{aligned}
$$

Для того чтобы снять ограничение (4), достаточно воспользоваться рассуждением из доказательства теоремы 2.4 в [27]. Таким образом, $C \lesssim D$.

ОПРЕДЕЛЕНИЕ. $\underline{v}(x):=\underset{|t|<|x|}{\operatorname{essinf}} v(t), v \in \mathfrak{M}^{+}$.

Теорема 2. Пусть $0<q<1, p=1$. Тогда наилучшая константа $C$ в неравенстве

$$
\left(\int_{\mathbb{R}^{n}}\left(\int_{B(|x|)} f\right)^{q} u(x) d x\right)^{\frac{1}{q}} \leq C \int_{\mathbb{R}^{n}} f v, \quad f \in \mathfrak{M}^{+},
$$

не изменится, если $v$ заменить на $\underline{v}$.

ДокАЗАтЕльСтво. Пусть $\underline{C}-$ наилучшая константа в неравенстве $(5)$ с $\underline{v}$ вместо $v$. Так как $\underline{v} \leq v$ п. в., то $C \leq \underline{C}$. Докажем обратное.

Фиксируем $f \in \mathfrak{M}^{+}$и $\varepsilon \in(0,1)$. Положим

$A:=\{x: v(x)<\varepsilon+\underline{v}(x)\}$,

$B_{j}:=\{x: j \varepsilon \leq \underline{v}(x)<(j+1) \varepsilon\} \subseteq\left\{x: a_{j} \leq|x| \leq b_{j}\right\}, j=0,1, \ldots$,

$a_{j}:=\inf B_{j}, b_{j}:=\sup B_{j}, J:=\left\{j: a_{j}<b_{j}\right\}$.

Тогда $\operatorname{mes}\left(\mathbb{R}^{n} \backslash \bigcup_{j \in J} B_{j}\right)=0$. Так как $\underline{v}$ невозрастающая, то

$$
\begin{gathered}
|x|<a_{j}, j \in J \Rightarrow \underline{v}(x) \geq \varepsilon(j+1) \Rightarrow \underline{v}\left(a_{j}\right) \geq \varepsilon(j+1), \\
|x|<b_{j}, j \in J \Rightarrow \underline{v}(x) \geq \varepsilon j \Rightarrow \underline{v}\left(b_{j}\right) \geq \varepsilon j,
\end{gathered}
$$

$B_{j}=\left\{x: a_{j}<|x| \leq b_{j}\right\}$. Имеем

$$
A_{j}:=A \cap\left\{x: a_{j}<|x|<a_{j}+\varepsilon\left(b_{j}-a_{j}\right)\right\} \subset B_{j} .
$$

Покажем, что $\operatorname{mes}\left(A_{j}\right)>0, j \in J$. Поскольку $v \geq \underline{v}$ п. в., то

$$
\begin{aligned}
\underline{v}\left(a_{j}\right)= & \underset{|t|<a_{j}}{\operatorname{essinf}} v(t) \geq(j+1) \varepsilon>\underline{v}\left(a_{j}+\varepsilon\left(b_{j}-a_{j}\right)\right)=\underset{|t|<a_{j}+\varepsilon\left(b_{j}-a_{j}\right)}{\operatorname{essinf}} v(t) \\
& =\min \left\{\underset{|t|<a_{j}}{\operatorname{essinf}} v(t), \underset{a_{j}<|t|<a_{j}+\varepsilon\left(b_{j}-a_{j}\right)}{\operatorname{essinf}} v(t)\right\}=\underset{a_{j}<|t|<a_{j}+\varepsilon\left(b_{j}-a_{j}\right)}{\operatorname{esinf}} v(t)=: z_{j} .
\end{aligned}
$$


По определению

$$
\underset{t \in E}{\operatorname{essinf}} v(t)=\sup \{c \in \mathbb{R}: \operatorname{mes}(t \in E: v(t)<c)=0\} .
$$

Тогда существует $c_{j} \in\left(z_{j},(j+1) \varepsilon\right)$ такое, что если $E_{j}:=\left\{t \in\left(z_{j},(j+1) \varepsilon\right)\right.$ : $\left.v(t)<c_{j}\right\}$, то $E_{j} \subset\left(a_{j}<|x|<a_{j}+\varepsilon\left(b_{j}-a_{j}\right)\right)$ и $\operatorname{mes}\left(E_{j}\right)>0$. Тем самым для любого $t \in E_{j}$ имеем

$$
v(t)<(j+1) \varepsilon \leq \underline{v}(t)+\varepsilon \Rightarrow E_{j} \subset A \Rightarrow \operatorname{mes}\left(A_{j}\right)>0 .
$$

Пусть $|A|:=\operatorname{mes} A$. Определим

$$
f_{\varepsilon}(x):=\sum_{j \in J}\left(\int_{a_{j}<|x|<b_{j}} f\right)\left|A_{j}\right|^{-1} \chi_{A_{j}}(x)
$$

В этом случае

$$
\begin{aligned}
\int_{\mathbb{R}^{n}} f_{\varepsilon} v=\sum_{j \in J}\left(\int_{a_{j}<|x|<b_{j}} f\right)\left|A_{j}\right|^{-1} \int_{A_{j}} v \leq \sum_{j \in J}\left(\int_{a_{j}<|x|<b_{j}} f\right)(j+2) \varepsilon \\
\quad \leq \sum_{j \in J}\left(\int_{a_{j}<|x|<b_{j}} f \underline{v}+2 \varepsilon \int_{a_{j}<|x|<b_{j}} f\right)=\iint_{\mathbb{R}^{n}} f \underline{v}+2 \varepsilon \int_{\mathbb{R}^{n}} f .
\end{aligned}
$$

Покажем, что $\int_{B(|x|)} f \leq \int_{B(|x|)} f_{\varepsilon}$ п. в. Так как $x \in \bigcup_{j \in J}\left(a_{j}+\varepsilon\left(b_{j}-a_{j}\right)<|x|<b_{j}\right) \Rightarrow \exists j_{0} \in J: x \in\left(a_{j 0}+\varepsilon\left(b_{j 0}-a_{j 0}\right)<|x|<b_{j 0}\right)$,

TO

$$
\begin{aligned}
\int_{B(|x|)} f \leq \sum_{j_{0} \leq j_{a_{j}<|x|<b_{j}}} \int_{j_{0} \leq j} f=\sum_{a_{j}<|x|<b_{j}}\left(\int_{a_{j}<|x|<a_{j}+\varepsilon\left(b_{j}-a_{j}\right)} f\right)\left|A_{j}\right|^{-1} & \chi_{A_{j}} \\
& =\sum_{j_{0} \leq j_{a_{j}<|x|<b_{j}}} \int_{\varepsilon} \leq \int_{B(|x|)} f_{\varepsilon} .
\end{aligned}
$$

Таким образом,

$$
\int_{B(|x|)} f \leq \liminf _{\varepsilon \rightarrow+0} \int_{B(|x|)} f_{\varepsilon}
$$

откуда, используя лемму Фату, получим

$$
\begin{aligned}
&\left(\int_{\mathbb{R}^{n}}\left(\int_{B(|x|)} f\right)^{q} u(x) d x\right)^{\frac{1}{q}} \leq \liminf _{\varepsilon \rightarrow+0}\left(\int_{\mathbb{R}^{n}}\left(\int_{B(|x|)} f_{\varepsilon}\right)^{q} u(x) d x\right)^{\frac{1}{q}} \\
& \leq \liminf _{\varepsilon \rightarrow+0} C \int_{\mathbb{R}^{n}} f_{\varepsilon} v \leq C \liminf _{\varepsilon \rightarrow+0}\left(\int_{\mathbb{R}^{n}} f \underline{v}+2 \varepsilon \int_{\mathbb{R}^{n}} f\right)=C \int_{\mathbb{R}^{n}} f \underline{v} .
\end{aligned}
$$

Таким образом, $\underline{C} \leq C$, и получаем $\underline{C}=C$. 
Теорема 3. Пусть $0<q<1, p=1$. Тогда для наилучшей константы $C$ в неравенстве (5) выполнено соотношение

$$
C \approx\left(\int_{\mathbb{R}^{n}} \underline{v}^{\frac{q}{q-1}}(x)\left(\int_{\mathbb{R}^{n} \backslash B(|x|)} u\right)^{\frac{q}{1-q}} u(x) d x\right)^{\frac{1-q}{q}}=: B .
$$

ДоказАтЕЛЬСтво. Из теоремы 2 следует, что неравенство (5) эквивалентно неравенству

$$
\left(\int_{\mathbb{R}^{n}}\left(\int_{B(|x|)} f\right)^{q} u(x) d x\right)^{\frac{1}{q}} \leq C\left(\int_{\mathbb{R}^{n}} f \underline{v}\right), \quad f \in \mathfrak{M}^{+},
$$

которое эквивалентно неравенству

$$
\begin{aligned}
\left(\int_{0}^{\infty} \int_{\Sigma_{n-1}} t^{n-1} u(t \tau) d \tau\left(\int_{0}^{t} \int_{\Sigma_{n-1}} s^{n-1} f(s \sigma) d s d \sigma\right)^{q} d t\right)^{\frac{1}{q}} \\
\leq C\left(\int_{0}^{\infty} \int_{\Sigma_{n-1}} t^{n-1} f(t \tau) \underline{v}(t) d t d \tau\right)
\end{aligned}
$$

Поскольку $\underline{v}(x)=\underline{v}(|x|)=\underline{v}(t)$ при $t=|x|$, имеем

$$
\left(\int_{0}^{\infty} \mathbf{u}(t)\left(\int_{0}^{t} \mathbf{f}(s) d s\right)^{q} d t\right)^{\frac{1}{q}} \leq C \int_{0}^{\infty} \mathbf{f} \underline{v} .
$$

Используя рассуждения теоремы 3.3 из [27], имеем

$$
\begin{gathered}
C \approx\left(\int_{0}^{\infty} \underline{v}^{\frac{q}{q-1}}(t)\left(\int_{t}^{\infty} \mathbf{u}\right)^{\frac{q}{1-q}} \mathbf{u}(t) d t\right)^{\frac{1-q}{q}} \\
=\left(\int_{0}^{\infty} \underline{v}^{\frac{q}{q-1}}(t) \int_{\Sigma_{n-1}} t^{n-1} u(t \tau) d \tau\left(\int_{t}^{\infty} \int_{\Sigma_{n-1}} s^{n-1} u(s \sigma) d s d \sigma\right)^{\frac{q}{1-q}} d t\right)^{\frac{1-q}{q}} \\
=\left(\int_{\mathbb{R}^{n}} \underline{v}^{\frac{q}{q-1}}(x)\left(\int_{\mathbb{R}^{n} \backslash B(|x|)} u\right)^{\frac{q}{1-q}} u(x) d x\right)^{\frac{1-q}{q}} .
\end{gathered}
$$

В дальнейшем для крайних значений параметров суммирования применяем критерии, которые находятся по общей теореме (см. [28, гл. $11 ; 29$, теорема 1.1]).

Лемма. Пусть $0<q \leq \infty, 1 \leq p \leq \infty$. Тогда для наилучшей константы $C$ в неравенстве (3) выполнены следующие соотношения:

(i) $1 \leq q<\infty, p=1, C=\sup _{x \in \mathbb{R}^{n}}\left(\int_{|y| \geq|x|} u(y) d y\right)^{\frac{1}{q}} \underset{|y|<|x|}{\operatorname{ess~sup}} v^{-1}(y)$;

(ii) $0<q<\infty, p=\infty, C=\left(\int_{\mathbb{R}^{n}}\left(\int_{|x| \leq|z|} v^{-1}(x) d x\right)^{q} u(z) d z\right)^{\frac{1}{q}}$ 
(iii) $1<p<\infty, q=1, C=\left(\int_{\mathbb{R}^{n}}\left(\int_{|x| \geq|z|} u(x) d x\right)^{p^{\prime}} v^{1-p^{\prime}}(z) d z\right)^{\frac{1}{p^{\prime}}}$;

(iv) $1<p<\infty, q=\infty, C=\underset{z \in \mathbb{R}^{n}}{\operatorname{ess} \sup } u(z)\left(\int_{|x| \leq|z|} v^{1-p^{\prime}}(x) d x\right)^{\frac{1}{p^{\prime}}}$;

(v) $p=q=\infty, C=\underset{z \in \mathbb{R}^{n}}{\operatorname{ess} u p} u(z)\left(\int_{|x| \leq|z|} v^{-1}(x) d x\right)$;

(vi) $p=1, q=\infty, C=\sup _{x \in \mathbb{R}^{n}} \operatorname{ess} \sup u(y) \underset{|y|}{\operatorname{ess} \sup v^{-1}(y)}$.

\section{§ 3. Многомерные билинейные неравенства Харди}

Для характеризации билинейного весового неравенства (2) разделим область значений параметров суммирования на следующие зоны.

$I_{1} . \quad 1<\max \left(p_{1}, p_{2}\right) \leq q<\infty$.

$I_{2} . \quad 1<\max \left(p_{1}, p_{2}\right)<q=\infty$.

$I_{3} . \quad q=\infty, p_{1}=p_{2}=\infty$.

$I I_{1} . \quad 1<\min \left(p_{1}, p_{2}\right) \leq q<\max \left(p_{1}, p_{2}\right)<\infty$.

$I I_{2} . \quad 1<\min \left(p_{1}, p_{2}\right) \leq q<\max \left(p_{1}, p_{2}\right)=\infty$.

$I I_{3} . \quad 0<\min \left(p_{1}, p_{2}\right)<q=\infty, \max \left(p_{1}, p_{2}\right)=\infty$.

$I I I_{1} . \quad 0<q<\min \left(p_{1}, p_{2}\right), \min \left(p_{1}, p_{2}\right)>1$.

$I I I_{2} . \quad 0<q<\min \left(p_{1}, p_{2}\right)<\infty, \min \left(p_{1}, p_{2}\right)>1, \max \left(p_{1}, p_{2}\right)=\infty$.

$I V_{1} . \quad 0<q<1, p_{1}=p_{2}=1$.

$I V_{2} . \quad 0<q<1=\min \left(p_{1}, p_{2}\right)<\infty, 1<\max \left(p_{1}, p_{2}\right)<\infty$.

$I V_{3} . \quad 0<q<1=\min \left(p_{1}, p_{2}\right), \max \left(p_{1}, p_{2}\right)=\infty$.

В дальнейшем будем использовать сокращения типа $\int_{|x| \leq \alpha} g:=\int_{|x| \leq \alpha} g(x) d x$.

Теорема 4 (случай $\left.I_{1}\right)$. Пусть $1<\max \left(p_{1}, p_{2}\right) \leq q<\infty$. Тогда $C \approx A_{1}$, где

$$
A_{1}:=\sup _{\alpha>0}\left(\int_{|x| \leq \alpha} v_{1}^{1-p_{1}^{\prime}}\right)^{\frac{1}{p_{1}^{\prime}}}\left(\int_{|x| \leq \alpha} v_{2}^{1-p_{2}^{\prime}}\right)^{\frac{1}{p_{2}^{\prime}}}\left(\int_{|x| \geq \alpha} u\right)^{\frac{1}{q}} .
$$

ДокАЗАТЕЛЬСтво. Дважды используя теорему 2.1 из [2], получим

$$
\begin{aligned}
C=\sup _{g} \sup _{f} \frac{\left(\int_{\mathbb{R}^{n}}\left(\int_{B(|x|)} f\right)^{q}\left(\int_{B(|x|)} g\right)^{q} u(x) d x\right)^{\frac{1}{q}}}{\|f\|_{p_{1}, v_{1}}\|g\|_{p_{2}, v_{2}}} \\
\approx \sup _{g}\|g\|_{p_{2}, v_{2}}^{-1} \sup _{\alpha>0}\left(\int_{|x| \geq \alpha}\left(\int_{B(|x|)} g\right)^{q} u(x) d x\right)^{\frac{1}{q}}\left(\int_{|x| \leq \alpha} v_{1}^{1-p_{1}^{\prime}}\right)^{\frac{1}{p_{1}^{\prime}}} \\
\quad \approx \sup _{\alpha>0}\left(\int_{|x| \leq \alpha} v_{1}^{1-p_{1}^{\prime}}\right)^{\frac{1}{p_{1}^{\prime}}} \sup _{\beta>\alpha}\left(\int_{|y| \geq \beta} u\right)^{\frac{1}{q}}\left(\int_{|y| \leq \beta} v_{2}^{1-p_{2}^{\prime}}\right)^{\frac{1}{p_{2}^{\prime}}}=A_{1} .
\end{aligned}
$$

Теорема 5 (случай $\left.I_{2}\right)$. Пусть $1<\max \left(p_{1}, p_{2}\right)<q=\infty$. Тогда $C \approx A_{2}$, где

$$
A_{2}:=\underset{x \in \mathbb{R}^{n}}{\operatorname{ess} \sup _{B}}\left(\int_{B(|x|)} v_{1}^{1-p_{1}^{\prime}}\right)^{\frac{1}{p_{1}^{\prime}}}\left(\int_{B(|x|)} v_{2}^{1-p_{2}^{\prime}}\right)^{\frac{1}{p_{2}^{\prime}}} u(x) .
$$

ДокАЗАТЕльСтво. Применяя лемму (iv) дважды, имеем $C \approx A_{2}$. 
Теорема 6 (случай $I_{3}$ ). Пусть $q=\infty, p_{1}=p_{2}=\infty$. Тогда $C \approx A_{3}$, где

$$
A_{3}:=\operatorname{ess~sup}_{x \in \mathbb{R}^{n}}\left(\int_{B(|x|)} v_{1}^{-1}\right)\left(\int_{B(|x|)} v_{2}^{-1}\right) u(x) .
$$

ДокАЗАТЕЛЬСтво. Применяя лемму (v) дважды, получим $C \approx A_{3}$.

Теорема 7 (случай $I I_{1}$ ). Пусть $1<\min \left(p_{1}, p_{2}\right) \leq q<\max \left(p_{1}, p_{2}\right)<\infty$ и $\frac{1}{r_{i}}:=\frac{1}{q}-\frac{1}{p_{i}}$. Тогда $C \approx A_{4}$, где

(a) $1<p_{1} \leq q<p_{2}<\infty$,

$$
A_{4}:=\sup _{\alpha>0}\left(\int_{|x| \leq \alpha} v_{1}^{1-p_{1}^{\prime}}\right)^{\frac{1}{p_{1}^{\prime}}}\left(\int_{|x| \geq \alpha}\left(\int_{|y| \geq|x|} u\right)^{\frac{r_{2}}{p_{2}}}\left(\int_{|y| \leq|x|} v_{2}^{1-p_{2}^{\prime}}\right)^{\frac{r_{2}}{p_{2}^{\prime}}} u(x) d x\right)^{\frac{1}{r_{2}}},
$$

(b) $1<p_{2} \leq q<p_{1}<\infty$,

$$
A_{4}:=\sup _{\alpha>0}\left(\int_{|x| \leq \alpha} v_{2}^{1-p_{2}^{\prime}}\right)^{\frac{1}{p_{2}^{\prime}}}\left(\int_{|x| \geq \alpha}\left(\int_{|y| \geq|x|} u\right)^{\frac{r_{1}}{p_{1}}}\left(\int_{|y| \leq|x|} v_{1}^{1-p_{1}^{\prime}}\right)^{\frac{r_{1}}{p_{1}^{\prime}}} u(x) d x\right)^{\frac{1}{r_{1}}} \text {. }
$$

ДокАЗАТЕЛЬСТво. Используя теорему 2.1 из [2] и теорему 1, получим

$$
\begin{aligned}
C=\sup _{g} \sup _{f} \frac{\left(\int_{\mathbb{R}^{n}}\left(\int_{B(|x|)} f\right)^{q}\left(\int_{B(|x|)} g\right)^{q} u(x) d x\right)^{\frac{1}{q}}}{\|f\|_{p_{1}, v_{1}}\|g\|_{p_{2}, v_{2}}} \\
\quad \approx \sup _{g}\|g\|_{p_{2}, v_{2}}^{-1} \sup _{\alpha>0}\left(\int_{|x| \geq \alpha}\left(\int_{B(|x|)} g\right)^{q} u(x) d x\right)^{\frac{1}{q}}\left(\int_{|x| \leq \alpha} v_{1}^{1-p_{1}^{\prime}}\right)^{\frac{1}{p_{1}^{\prime}}} \\
\approx \sup _{\alpha>0}\left(\int_{|x| \leq \alpha} v_{1}^{1-p_{1}^{\prime}}\right)^{\frac{1}{p_{1}^{\prime}}}\left(\int_{|x| \geq \alpha}\left(\int_{|y| \geq|x|} u\right)^{\frac{r_{2}}{p_{2}}}\left(\int_{|y| \leq|x|} v_{2}^{1-p_{2}^{\prime}}\right)^{\frac{r_{2}}{p_{2}^{\prime}}} u(x) d x\right)^{\frac{1}{r_{2}}} .
\end{aligned}
$$

Случай $1<p_{2} \leq q<p_{1}<\infty$ доказывается аналогично.

Теорема 8 (случай $\left.I I_{2}\right)$. Пусть $1<\min \left(p_{1}, p_{2}\right) \leq q<\max \left(p_{1}, p_{2}\right)=\infty$. Тогда $C \approx A_{5}$, где

(a) $1<p_{1} \leq q<p_{2}=\infty$,

$$
A_{5}:=\sup _{\alpha>0}\left(\int_{|x| \leq \alpha} v_{1}^{1-p_{1}^{\prime}}\right)^{\frac{1}{p_{1}^{\prime}}}\left(\int_{|x| \geq \alpha} u(x)\left(\int_{|y|<|x|} v_{2}^{-1}\right)^{q} d x\right)^{\frac{1}{q}},
$$

(b) $1<p_{2} \leq q<p_{1}=\infty$,

$$
A_{5}:=\sup _{\alpha>0}\left(\int_{|x| \leq \alpha} v_{2}^{1-p_{2}^{\prime}}\right)^{\frac{1}{p_{2}^{\prime}}}\left(\int_{|x| \geq \alpha} u(x)\left(\int_{|y|<|x|} v_{1}^{-1}\right)^{q} d x\right)^{\frac{1}{q}} .
$$

ДокАЗАТЕЛЬСтво. Применение теоремы 2.1 из [2] и леммы (ii) влечет $C \approx$ $A_{5}$. 
Теорема 9 (случай $\left.I I_{3}\right)$. Пусть $1<\min \left(p_{1}, p_{2}\right)<q=\infty, \max \left(p_{1}, p_{2}\right)=\infty$. Тогда $C \approx A_{6}$, где

(a) $1<p_{1}<q=p_{2}=\infty$,

$$
A_{6}:=\operatorname{esssup}_{x \in \mathbb{R}^{n}}\left(\int_{B(|x|)} v_{1}^{1-p_{1}^{\prime}}\right)^{\frac{1}{p_{1}^{\prime}}}\left(\int_{B(|x|)} v_{2}^{-1}\right) u(x),
$$

(b) $1<p_{2} \leq q<p_{1}=\infty$,

$$
A_{6}:=\operatorname{essup}_{x \in \mathbb{R}^{n}}\left(\int_{B(|x|)} v_{2}^{1-p_{2}^{\prime}}\right)^{\frac{1}{p_{2}^{\prime}}}\left(\int_{B(|x|)} v_{1}^{-1}\right) u(x) .
$$

ДоКАЗАТЕЛЬСТво. Применяя лемму в случаях (iv) и (v), получим $C \approx$ $A_{6}$.

Теорема 10 (случай $\left.I I I_{1}\right)$. Пусть $0<q<\min \left(p_{1}, p_{2}\right)<\infty, \min \left(p_{1}, p_{2}\right)>1$ и $\frac{1}{r_{i}}:=\frac{1}{q}-\frac{1}{p_{i}}, i=1,2$. Тогда $C \approx A_{7.1}+A_{7.2}$, где

(a) $\frac{1}{q} \leq \frac{1}{p_{1}}+\frac{1}{p_{2}}$,

$$
\begin{aligned}
& A_{7.1}:=\sup _{\alpha>0}\left(\int_{|x| \leq \alpha} v_{1}^{1-p_{1}^{\prime}}\right)^{\frac{1}{p_{1}^{\prime}}}\left(\int_{|x| \geq \alpha}\left(\int_{\mathbb{R}^{n} \backslash B(|x|)} u\right)^{\frac{r_{2}}{q}}\left(\int_{B(|x|)} v_{2}^{1-p_{2}^{\prime}}\right)^{\frac{r_{2}}{q^{\prime}}} v_{2}^{1-p_{2}^{\prime}}(x) d x\right)^{\frac{1}{r_{2}}}, \\
& A_{7.2}:=\sup _{\alpha>0}\left(\int_{|x| \leq \alpha} v_{2}^{1-p_{2}^{\prime}}\right)^{\frac{1}{p_{2}^{\prime}}}\left(\int_{|x| \geq \alpha}\left(\int_{\mathbb{R}^{n} \backslash B(|x|)} u\right)^{\frac{r_{1}}{q}}\left(\int_{B(|x|)} v_{1}^{1-p_{1}^{\prime}}\right)^{\frac{r_{1}}{q^{\prime}}} v_{1}^{1-p_{1}^{\prime}}(x) d x\right)^{\frac{1}{r_{1}}},
\end{aligned}
$$

(b) $\frac{1}{q}>\frac{1}{p_{1}}+\frac{1}{p_{2}}, \frac{1}{s}:=\frac{1}{q}-\frac{1}{p_{1}}-\frac{1}{p_{2}}$,

$$
\begin{aligned}
A_{7.1}^{s}:=\int_{\mathbb{R}^{n}}\left(\int_{\mathbb{R}^{n} \backslash B(|x|)}\left(\int_{\mathbb{R}^{n} \backslash B(|y|)} u\right)^{\frac{r_{2}}{q}}\left(\int_{B(|x|)} v_{2}^{1-p_{2}^{\prime}}\right)^{\frac{r_{2}}{q^{\prime}}} v_{2}^{1-p_{2}^{\prime}}(y) d y\right)^{\frac{s}{r_{2}}} \\
\times\left(\int_{B(|x|)} v_{1}^{1-p_{1}^{\prime}}\right)^{\frac{s}{r_{2}^{\prime}}} v_{1}^{1-p_{1}^{\prime}}(x) d x, \\
A_{7.2}^{s}:=\int_{\mathbb{R}^{n}}\left(\int_{\mathbb{R}^{n} \backslash B(|x|)}\left(\int_{\mathbb{R}^{n} \backslash B(|y|)} u\right)^{\frac{r_{1}}{q}}\left(\int_{B(|x|)} v_{1}^{1-p_{1}^{\prime}}\right)^{\frac{r_{1}}{q^{\prime}}} v_{1}^{1-p_{1}^{\prime}}(y) d y\right)^{\frac{s}{r_{1}}} \\
\times\left(\int_{B(|x|)} v_{2}^{1-p_{2}^{\prime}}\right)^{\frac{s}{r_{1}^{\prime}}} v_{2}^{1-p_{2}^{\prime}}(x) d x .
\end{aligned}
$$

ДокАЗАТЕЛЬСтво. (а) Применяя теорему 1, неравенство Гёльдера и теорему Фубини, получим

$$
C=\sup _{g} \sup _{f} \frac{\left(\int_{\mathbb{R}^{n}}\left(\int_{B(|x|)} f\right)^{q}\left(\int_{B(|x|)} g\right)^{q} u(x) d x\right)^{\frac{1}{q}}}{\|f\|_{p_{1}, v_{1}}\|g\|_{p_{2}, v_{2}}} \approx \sup _{g}\|g\|_{p_{2}, v_{2}}^{-1}
$$




$$
\begin{gathered}
\left.\times\left(\int_{\mathbb{R}^{n}} \int_{\mathbb{R}^{n} \backslash B(|x|)}\left(\int_{B(|y|)} g\right)^{q} u(y) d y\right)^{\frac{r_{1}}{q}}\left(\int_{B(|x|)} v_{1}^{1-p_{1}^{\prime}}\right)^{\frac{r_{1}}{q^{\prime}}} v_{1}^{1-p_{1}^{\prime}}(x) d x\right)^{\frac{1}{r_{1}}} \\
=\sup _{g}\|g\|_{p_{2}, v_{2}}^{-1}\left[\sup _{h} \frac{\int_{\mathbb{R}^{n}}\left(\int_{B(|x|)} g\right)^{q}\left(\int_{B(|x|)} h\right) u(x) d x}{\left(\int_{\mathbb{R}^{n}} h^{\frac{p_{1}}{q}}(x)\left(\int_{B(|x|)} v_{1}^{1-p_{1}^{\prime}}\right)^{\frac{-p_{1}}{q^{\prime}}} v_{1}^{\frac{p_{1}^{\prime}}{r_{1}}}(x) d x\right)^{\frac{q}{p_{1}}}}\right] \\
\approx \sup _{h} \frac{\left(\int_{\mathbb{R}^{n}}\left(\int_{\mathbb{R}^{n} \backslash B(|x|)}\left(\int_{B(|y|)} h\right) u(y) d y\right)^{\frac{r_{2}}{q}}\left(\int_{B(|x|)} v_{2}^{1-p_{2}^{\prime}}\right)^{\frac{r_{2}}{q^{\prime}}} v_{2}^{1-p_{2}^{\prime}}(x) d x\right)^{\frac{1}{r_{2}}}}{\left(\int_{\mathbb{R}^{n}} h^{\frac{p_{1}}{q}}(x)\left(\int_{B(|x|)} v_{1}^{1-p_{1}^{\prime}}\right)^{\frac{-p_{1}}{q^{\prime}}} v_{1}^{\frac{p_{1}^{\prime}}{r_{1}}}(x) d x\right)^{\frac{1}{p_{1}}}} \\
\approx \sup _{h} \frac{\left(\int_{\mathbb{R}^{n}}\left(\int_{B(|x|)} h\right)^{\frac{r_{2}}{q}}\left(\int_{\mathbb{R}^{n} \backslash B(|x|)} u\right)^{\frac{r_{2}}{q}}\left(\int_{B(|x|)} v_{2}^{1-p_{2}^{\prime}}\right)^{\frac{r_{2}}{q^{\prime}}} v_{2}^{1-p_{2}^{\prime}}(x) d x\right)^{\frac{1}{r_{2}}}}{\left(\int_{\mathbb{R}^{n}} h^{\frac{p_{1}}{q}}(x)\left(\int_{B(|x|)} v_{1}^{1-p_{1}^{\prime}}\right)^{\frac{-p_{1}}{q^{\prime}}} v_{1}^{\frac{p_{1}^{\prime}}{r_{1}}}(x) d x\right)^{\frac{1}{p_{1}}}} \\
+\sup _{h} \frac{\left(\int_{\mathbb{R}^{n}}\left(\int_{\mathbb{R}^{n} \backslash B(|x|)} h\right)^{\frac{r_{2}}{q}}\left(\int_{B(|x|)} v_{2}^{1-p_{2}^{\prime}}\right)^{\frac{r_{2}}{q^{\prime}}} v_{2}^{1-p_{2}^{\prime}}(x) d x\right)^{\frac{1}{r_{2}}}}{\left(\int_{\mathbb{R}^{n}} h^{\frac{p_{1}}{q}}(x)\left(\int_{\mathbb{R}^{n} \backslash B(|x|)} u\right)^{\frac{-p_{1}}{q}}\left(\int_{B(|x|)} v_{1}^{1-p_{1}^{\prime}}\right)^{\frac{-p_{1}}{q^{\prime}}} v_{1}^{\frac{p_{1}^{\prime}}{r_{1}}}(x) d x\right)^{\frac{1}{p_{1}}}}=: H_{1}+H_{2} .
\end{gathered}
$$

Для оценки констант $H_{1}$ и $H_{2}$ применим теорему 2.1 из [2] с параметрами $\tilde{p} \leq \tilde{q}$, где $\tilde{p}=\frac{p_{1}}{q}, \tilde{q}=\frac{r_{2}}{q}$, и получим $H_{1} \approx A_{7.1}$ и $H_{2} \approx A_{7.2}$.

П. (b) доказывается аналогично применением теоремы 1.

Теорема 11 (случай $\left.I I I_{2}\right)$. Пусть $0<q<\min \left(p_{1}, p_{2}\right)<\infty, \min \left(p_{1}, p_{2}\right)>1$, $\max \left(p_{1}, p_{2}\right)=\infty$ и $\frac{1}{r_{i}}:=\frac{1}{q}-\frac{1}{p_{i}}, i=1,2$. Тогда $C \approx A_{8}$, где

(a) $0<q<p_{1}<\infty, p_{1}>1, p_{2}=\infty$,

$$
\left.A_{8}:=\int_{\mathbb{R}^{n}}\left(\int_{B(|x|)} v_{1}^{1-p_{1}^{\prime}}\right)^{\frac{r_{1}}{q^{\prime}}} v_{1}^{1-p_{1}^{\prime}}(x)\left(\int_{\mathbb{R}^{n} \backslash B(|x|)} u(y)\left(\int_{B(|y|)} v_{2}^{-1}\right)^{q} d y\right)^{\frac{r_{1}}{q}} d x\right)^{\frac{1}{r_{1}}},
$$

(b) $0<q<p_{2}<\infty, p_{2}>1, p_{1}=\infty$,

$$
A_{8}:=\left(\int_{\mathbb{R}^{n}}\left(\int_{B(|x|)} v_{2}^{1-p_{2}^{\prime}}\right)^{\frac{r_{2}}{q^{\prime}}} v_{2}^{1-p_{2}^{\prime}}(x)\left(\int_{\mathbb{R}^{n} \backslash B(|x|)} u(y)\left(\int_{B(|y|)} v_{1}^{-1}\right)^{q} d y\right)^{\frac{r_{2}}{q}} d x\right)^{\frac{1}{r_{2}}} .
$$

ДокАзАтЕЛЬСтво. (а) Применяя теорему 1 , принцип двойственности и утверждения леммы (ii) и (iii), получим

$$
\begin{aligned}
C= & \sup _{g} \sup _{f} \frac{\left(\int_{\mathbb{R}^{n}}\left(\int_{B(|x|)} f\right)^{q}\left(\int_{B(|x|)} g\right)^{q} u(x) d x\right)^{\frac{1}{q}}}{\|f\|_{p_{1}, v_{1}}\|g\|_{p_{2}, v_{2}}} \approx \sup _{g}\|g\|_{p_{2}, v_{2}}^{-1} \\
& \times\left(\int_{\mathbb{R}^{n}}\left(\int_{\mathbb{R}^{n} \backslash B(|x|)}\left(\int_{B(|y|)} g\right)^{q} u(y) d y\right)^{\frac{r_{1}}{q}}\left(\int_{B(|x|)} v_{1}^{1-p_{1}^{\prime}}\right)^{\frac{r_{1}}{q^{\prime}}} v_{1}^{1-p_{1}^{\prime}}(x) d x\right)^{\frac{1}{r_{1}}}
\end{aligned}
$$




$$
\begin{gathered}
=\sup _{g}\|g\|_{p_{2}, v_{2}}^{-1}\left[\sup _{h} \frac{\int_{\mathbb{R}^{n}}\left(\int_{B(|x|)} g\right)^{q}\left(\int_{B(|x|)} h\right) u(x) d x}{\left(\int_{\mathbb{R}^{n}} h^{\frac{p_{1}}{q}}(x)\left(\int_{B(|x|)} v_{1}^{1-p_{1}^{\prime}}\right)^{\frac{-p_{1}}{q^{\prime}}} v_{1}^{\frac{p_{1}^{\prime}}{r_{1}}}(x) d x\right)^{\frac{q}{p_{1}}}}\right]^{\frac{1}{q}} \\
\left.=\sup _{h}\left[\frac{\int_{\mathbb{R}^{n}}\left(\int_{B(|x|)} h\right)\left(\int_{B(|x|)} v_{2}^{-1}\right)^{q} u(x) d x}{\left(\int_{\mathbb{R}^{n}} h^{\frac{p_{1}}{q}}(x)\left(\int_{B(|x|)} v_{1}^{1-p_{1}^{\prime}}\right)^{\frac{-p_{1}}{q^{\prime}}} v_{1}^{\frac{p_{1}^{\prime}}{r_{1}}}(x) d x\right)^{\frac{q}{p_{1}}}}\right]^{\frac{r_{1}}{q^{\prime}}} v_{1}^{1-p_{1}^{\prime}}(x)\left(\int_{\mathbb{R}^{n} \backslash B(|x|)} u(y)\left(\int_{B(|y|)} v_{2}^{-1}\right)^{q} d y\right)^{\frac{r_{1}}{q}} d x\right)^{\frac{1}{r_{1}}} .
\end{gathered}
$$

Случай (b) доказывается аналогично.

Пусть $v_{i} \in \mathfrak{M}^{+}, i=1,2$. Положим

$$
\underline{v}_{i}(x):=\underset{|t|<|x|}{\operatorname{essinf}} v_{i}(t)=\frac{1}{\underset{|t|<|x|}{\operatorname{ess} \sup } \frac{1}{v_{i}(t)}}=: \frac{1}{\overline{v_{i}}(t)} .
$$

Заметим, что $\underline{v}_{i}(x)=\underline{v}_{i}(|x|)$ и $\bar{v}_{i}(x)=\bar{v}_{i}(|x|)$, будем использовать эти обозначения для функций $\bar{v}_{i}(t):=\bar{v}_{i}(|x|), t=|x|$ при $t \in[0, \infty)$. Без потери общности предположим, что функция $\bar{v}_{i}(t)$ непрерывна справа. Тогда существует мера Бореля $d \varphi_{i}$ такая, что

$$
\left[\bar{v}_{i}(t)\right]^{\frac{q}{1-q}}=\int_{[0, t]} d \varphi_{i}+\left[\bar{v}_{i}(0)\right]^{\frac{q}{1-q}} .
$$

Рассмотрим неравенство

$$
\left(\int_{\mathbb{R}^{n}}\left(\int_{B(|x|)} f\right)^{q}\left(\int_{B(|x|)} g\right)^{q} u(x) d x\right)^{\frac{1}{q}} \leq C\left(\int_{\mathbb{R}^{n}} f v_{1}\right)\left(\int_{\mathbb{R}^{n}} g v_{2}\right), \quad f, g \in \mathfrak{M}^{+},
$$

и предположим для простоты, что $\bar{v}_{i}(0)=\lim _{t \rightarrow 0+0} \bar{v}_{i}(t)=0, i=1,2$.

Теорема 12 (случай $I V_{1}$ ). Пусть $0<q<1, p_{1}=p_{2}=1$. Тогда $C \approx$ $A_{9.1}+A_{9.2}$, где

(a) $\frac{1}{2} \leq q<1$,

$$
\begin{aligned}
& A_{9.1}:=\sup _{t>0}\left(\int_{|x|>t}\left(\int_{|y|>|x|} u\right)^{\frac{q}{1-q}} u(x)\left(\left[\bar{v}_{2}(|x|)\right]^{\frac{q}{1-q}}-\left[\bar{v}_{2}(t)\right]^{\frac{q}{1-q}}\right) d x\right)^{\frac{1-q}{q}} \bar{v}_{1}(t), \\
& A_{9.2}:=\sup _{t>0}\left(\int_{|x|>t}\left(\int_{|y|>|x|} u\right)^{\frac{q}{1-q}} u(x)\left(\left[\bar{v}_{1}(|x|)\right]^{\frac{q}{1-q}}-\left[\bar{v}_{1}(t)\right]^{\frac{q}{1-q}}\right) d x\right)^{\frac{1-q}{q}} \bar{v}_{2}(t),
\end{aligned}
$$

(b) $0<q<\frac{1}{2}$,

$$
\begin{array}{r}
A_{9.1}^{\frac{q}{1-2 q}}:=\int_{\mathbb{R}^{n}}\left(\int_{\mathbb{R}^{n} \backslash B(|x|)}\left(\int_{\mathbb{R}^{n} \backslash B(|y|)} u\right)^{\frac{q}{1-q}} u(y) d y\left(\left[\bar{v}_{2}(|y|)\right]^{\frac{q}{1-q}}-\left[\bar{v}_{2}(|x|)\right]^{\frac{q}{1-q}}\right)\right)^{\frac{1-q}{1-2 q}} \\
\times d\left[\bar{v}_{1}(|x|)\right]^{\frac{q}{1-2 q}}
\end{array}
$$




$$
\begin{array}{r}
A_{9.2}^{\frac{q}{1-2 q}}:=\int_{\mathbb{R}^{n}}\left(\int_{\mathbb{R}^{n} \backslash B(|x|)}\left(\int_{\mathbb{R}^{n} \backslash B(|y|)} u\right)^{\frac{q}{1-q}} u(y) d y\left(\left[\bar{v}_{1}(|y|)\right]^{\frac{q}{1-q}}-\left[\bar{v}_{1}(|x|)\right]^{\frac{q}{1-q}}\right)\right)^{\frac{1-q}{1-2 q}} \\
\times d\left[\bar{v}_{2}(|x|)\right]^{\frac{q}{1-2 q}} .
\end{array}
$$

ДокАЗАтеЛЬСтво. (а) Введем обозначения, пусть

$$
\mathbf{h}(t):=\int_{\Sigma_{n-1}} t^{n-1} h(t \tau) d \tau,
$$

и аналогично определим функции $\mathbf{u}$ и g. Воспользовавшись теоремой 3 , неравенством Гёльдера и теоремой 3.3 из [27], получим

$$
\begin{aligned}
& C=\sup _{g} \sup _{f} \frac{\left(\int_{\mathbb{R}^{n}}\left(\int_{B(|x|)} f\right)^{q}\left(\int_{B(|x|)} g\right)^{q} u(x) d x\right)^{\frac{1}{q}}}{\|f\|_{1, v_{1}}\|g\|_{1, v_{2}}} \\
& \approx \sup _{g}\|g\|_{1, v_{2}}^{-1}\left(\int_{\mathbb{R}^{n}} \bar{v}_{1}^{\frac{q}{1-q}}(x)\left(\int_{\mathbb{R}^{n} \backslash B(|x|)}\left(\int_{B(|y|)} g\right)^{q} u(y) d y\right)^{\frac{q}{1-q}}\left(\int_{B(|x|)} g\right)^{q} u(x) d x\right)^{\frac{1-q}{q}} \\
& =\sup _{g}\|g\|_{1, v_{2}}^{-1}\left(\int_{0}^{\infty} \bar{v}_{1}^{\frac{q}{1-q}}(t)\left(\int_{t}^{\infty}\left(\int_{0}^{s} \mathbf{g}\right)^{q} \mathbf{u}(s) d s\right)^{\frac{q}{1-q}}\left(\int_{0}^{t} \mathbf{g}\right)^{q} \mathbf{u}(t) d t\right)^{\frac{1-q}{q}} \\
& \approx \sup _{g}\|g\|_{1, v_{2}}^{-1}\left(\int_{[0, \infty]}\left(\int_{t}^{\infty}\left(\int_{0}^{s} \mathbf{g}\right)^{q} \mathbf{u}(s) d s\right)^{\frac{1}{1-q}} d \varphi_{1}(t)\right)^{\frac{1-q}{q}} \\
& =\sup _{g}\|g\|_{1, v_{2}}^{-1}\left[\sup _{h} \frac{\int_{0}^{\infty}\left(\int_{0}^{t} \mathbf{g}\right)^{q}\left(\int_{[0, t]} \mathbf{h} d \varphi_{1}\right) \mathbf{u}(t) d t}{\left(\int_{[0, \infty]} \mathbf{h}^{\frac{1}{q}} d \varphi_{1}\right)^{q}}\right]^{\frac{1}{q}} \\
& \approx \sup _{h} \frac{\left(\int_{0}^{\infty} \bar{v}_{2}^{\frac{q}{1-q}}(t)\left(\int_{t}^{\infty}\left(\int_{[0, s]} \mathbf{h} d \varphi_{1}\right) \mathbf{u}(s) d s\right)^{\frac{q}{1-q}}\left(\int_{[0, t]} \mathbf{h} d \varphi_{1}\right) \mathbf{u}(t) d t\right)^{\frac{1-q}{q}}}{\int_{[0, \infty]} \mathbf{h}^{\frac{1}{q}} d \varphi_{1}} \\
& =\sup _{h} \frac{\left(\int_{[0, \infty]}\left(\int_{t}^{\infty}\left(\int_{[0, s]} \mathbf{h} d \varphi_{1}\right) \mathbf{u}(s) d s\right)^{\frac{1}{1-q}} d \varphi_{2}(t)\right)^{\frac{1-q}{q}}}{\int_{[0, \infty]} \mathbf{h}^{\frac{1}{q}} d \varphi_{1}}
\end{aligned}
$$

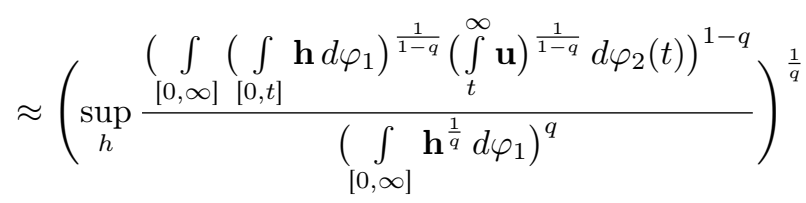

$$
\begin{aligned}
& +\left(\sup _{h} \frac{\left(\int 0, \infty\right][t, \infty]}{\left.\left(\iint_{s} \mathbf{h}(s)\left(\int_{s}^{\infty} \mathbf{u}\right) d \varphi_{1}(s)\right)^{\frac{1}{1-q}} d \varphi_{2}(t)\right)^{1-q}}\right)^{\frac{1}{q}}=: H_{1}^{\frac{1}{q}}+H_{2}^{\frac{1}{q}} .
\end{aligned}
$$


Чтобы оценить константы $H_{1}$ и $H_{2}$, применим теорему 1 из [30] при $\frac{1}{q} \leq \frac{1}{1-q} \Leftrightarrow$ $\frac{1}{2} \leq q<1$. Имеем

$$
\begin{aligned}
H_{1} \approx \sup _{t>0}\left(\int_{t, \infty]}\left(\int_{s}^{\infty} \mathbf{u}\right)^{\frac{1}{1-q}} d \varphi_{2}(s)\right)^{1-q}\left(\int_{[0, t]} d \varphi_{1}\right)^{1-q} \\
\quad \approx \sup _{t>0}\left(\int_{t, \infty]}\left(\int_{s}^{\infty}\left(\int_{z}^{\infty} \mathbf{u}\right)^{\frac{q}{1-q}} \mathbf{u}(z) d z\right) d \varphi_{2}(s)\right)^{1-q}\left(\bar{v}_{1}(t)\right)^{q} \\
=\sup _{t>0}\left(\int_{t}^{\infty}\left(\int_{z}^{\infty} \mathbf{u}\right)^{\frac{q}{1-q}} \mathbf{u}(z) d z\left(\int_{[t, z]} d \varphi_{2}\right)\right)^{1-q}\left(\bar{v}_{1}(t)\right)^{q} \\
=\sup _{t>0}\left(\int_{|x|>t}\left(\int_{|y|>|x|} u\right)^{\frac{q}{1-q}} u(x)\left(\left[\bar{v}_{2}(|x|)\right]^{\frac{q}{1-q}}-\left[\bar{v}_{2}(t)\right]^{\frac{q}{1-q}}\right) d x\right)^{1-q}\left(\bar{v}_{1}(t)\right)^{q} .
\end{aligned}
$$

Аналогично

$$
H_{2} \approx \sup _{t>0}\left(\int_{|x|>t}\left(\int_{|y|>|x|} u\right)^{\frac{q}{1-q}} u(x)\left(\left[\bar{v}_{1}(|x|)\right]^{\frac{q}{1-q}}-\left[\bar{v}_{1}(t)\right]^{\frac{q}{1-q}}\right) d x\right)^{1-q}\left(\bar{v}_{2}(t)\right)^{q} .
$$

(b) Используя те же рассуждения, получим

$$
\begin{aligned}
C=\sup _{g} \sup _{f} \frac{\left(\int_{\mathbb{R}^{n}}\left(\int_{B(|x|)} f\right)^{q}\left(\int_{B(|x|)} g\right)^{q} u(x) d x\right)^{\frac{1}{q}}}{\|f\|_{1, v_{1}}\|g\|_{1, v_{2}}} \\
\approx\left(\sup _{h} \frac{\left(\int[0, \infty]\left[\int_{0, t]} \mathbf{h} d \varphi_{1}\right)^{\frac{1}{1-q}}\left(\int_{t}^{\infty} \mathbf{u}\right)^{\frac{1}{1-q}} d \varphi_{2}(t)\right)^{1-q}}{\left(\int_{[0, \infty]} \mathbf{h}^{\frac{1}{q}} d \varphi_{1}\right)^{q}}\right. \\
+\left(\sup _{h} \frac{\left(\int_{[0, \infty][t, \infty]}\left(\int_{s}^{\frac{1}{q}} \mathbf{h}(s)\left(\int_{s}^{\infty} \mathbf{u}\right) d \varphi_{1}(s)\right)^{\frac{1}{1-q}} d \varphi_{2}(t)\right)^{1-q}}{\left(\int_{[0, \infty]} \mathbf{h}^{\frac{1}{q}} d \varphi_{1}\right)^{q}}=: H_{1}^{\frac{1}{q}}+H_{2}^{\frac{1}{q}} .\right.
\end{aligned}
$$

Для оценки констант $H_{1}$ и $H_{2}$ применим теорему 2 из [30] при $\frac{1}{1-q}<\frac{1}{q} \Leftrightarrow 0<$ $q<\frac{1}{2}$. Имеем

$$
\begin{aligned}
& H_{1}^{\frac{1}{1-2 q}} \approx \int_{[0, \infty]}\left(\int_{[0, t]} d \varphi_{1}\right)^{\frac{1-q}{1-2 q}}\left(\int_{[t, \infty]}\left(\int_{s}^{\infty} \mathbf{u}\right)^{\frac{1}{1-q}} d \varphi_{2}(s)\right)^{\frac{q}{1-2 q}}\left(\int_{t}^{\infty} \mathbf{u}\right)^{\frac{1}{1-q}} d \varphi_{2}(t) \\
&=\iint_{[0, \infty]}\left(\int_{[0, t]} d \varphi_{1}\right)^{\frac{q}{1-2 q}} d \varphi_{1}(s)\left(\int_{[t, \infty]}\left(\int_{s}^{\infty} \mathbf{u}\right)^{\frac{1}{1-q}} d \varphi_{2}(s)\right)^{\frac{q}{1-2 q}} \\
& \times\left(\int_{t}^{\infty} \mathbf{u}\right)^{\frac{1}{1-q}} d \varphi_{2}(t) \approx \int_{[0, \infty]}\left(\int_{t, \infty]}\left(\int_{s}^{\infty} \mathbf{u}\right)^{\frac{1}{1-q}} d \varphi_{2}(s)\right)^{\frac{1-q}{1-2 q}}\left(\int_{[0, t]} d \varphi_{1}\right)^{\frac{q}{1-2 q}} d \varphi_{1}(t)
\end{aligned}
$$




$$
\begin{array}{r}
=\int_{[0, \infty]}\left(\int_{t}^{\infty}\left(\int_{z}^{\infty} \mathbf{u}\right)^{\frac{q}{1-q}} \mathbf{u}(z) d z\left(\int_{[t, z]} d \varphi_{2}\right)\right)^{\frac{1-q}{1-2 q}} d\left[\bar{v}_{1}(t)\right]^{\frac{q}{1-2 q}} \\
=\int_{\mathbb{R}^{n}}\left(\int_{\mathbb{R}^{n} \backslash B(|x|)}\left(\int_{\mathbb{R}^{n} \backslash B(|y|)} u\right)^{\frac{q}{1-q}} u(y) d y\left(\left[\bar{v}_{2}(|y|)\right]^{\frac{q}{1-q}}-\left[\bar{v}_{2}(|x|)\right]^{\frac{q}{1-q}}\right)\right)^{\frac{1-q}{1-2 q}} \\
\times d\left[\bar{v}_{1}(|x|)\right]^{\frac{q}{1-2 q}} .
\end{array}
$$

Аналогично

$$
\begin{aligned}
H_{2}^{\frac{1}{1-2 q}} \approx \int_{\mathbb{R}^{n}}\left(\int _ { \mathbb { R } ^ { n } \backslash B ( | x | ) } ( \int _ { \mathbb { R } ^ { n } \backslash B ( | y | ) } u ) ^ { \frac { q } { 1 - q } } u ( y ) d y \left(\left[\bar{v}_{1}(|y|)\right]^{\frac{q}{1-q}}-\right.\right. & {\left.\left.\left[\bar{v}_{1}(|x|)\right]^{\frac{q}{1-q}}\right)\right)^{\frac{1-q}{1-2 q}} } \\
& \times d\left[\bar{v}_{2}(|x|)\right]^{\frac{q}{1-2 q}} .
\end{aligned}
$$

Теорема 13 (случай $\left.I V_{2}\right)$. Пусть $0<q<1=\min \left(p_{1}, p_{2}\right), 1<\max \left(p_{1}, p_{2}\right)<$ $\infty$ и $\frac{1}{r_{i}}:=\frac{1}{q}-\frac{1}{p_{i}}, i=1,2$. Тогда $C \approx A_{10.1}+A_{10.2}$, где

(a) $0<q<1=p_{1}, p_{2}>1, \frac{1}{s}=\frac{1}{q}-\frac{1}{p_{2}}-1, r_{2} \geq 1$,

$$
\begin{aligned}
& A_{10.1}:=\sup _{t>0}\left(\int_{|x|>t}\left(\int_{|y|>|x|} u\right)^{\frac{r_{2}}{q}}\left(\int_{|y|<|x|} v_{2}^{1-p_{2}^{\prime}}\right)^{\frac{r_{2}}{q^{\prime}}} v_{2}^{1-p_{2}^{\prime}}(x) d x\right)^{\frac{1}{r_{2}}} \bar{v}_{1}(t), \\
& A_{10.2}:=\sup _{t>0}\left(\int_{|x|>t}\left(\int_{|y|>|x|} u\right)^{\frac{q}{1-q}} u(x)\left(\left[\bar{v}_{1}(|x|)\right]^{\frac{q}{1-q}}-\left[\bar{v}_{1}(t)\right]^{\frac{q}{1-q}}\right) d x\right)^{\frac{1-q}{q}} \\
& \times\left(\int_{|x|<t} v_{2}^{1-p_{2}^{\prime}}\right)^{\frac{1}{p_{2}^{\prime}}} ;
\end{aligned}
$$

$r_{2}<1$,

$$
\begin{aligned}
& A_{10.1}:=\left(\int_{[0, \infty]}\left(\int_{|x|>t}\left(\int_{|y|>|x|} u\right)^{\frac{r_{2}}{q}}\left(\int_{|y|<|x|)} v_{2}^{1-p_{2}^{\prime}}\right)^{\frac{r_{2}}{q^{\prime}}} v_{2}^{1-p_{2}^{\prime}}(x) d x\right)^{\frac{s}{r_{2}}} d \bar{v}_{1}^{s}(t)\right)^{\frac{1}{s}}, \\
& A_{10.2}^{s}:=\int_{[0, \infty]}\left(\int_{|x|>t}\left(\int_{|y|>|x|} u\right)^{\frac{q}{1-q}} u(x)\left(\left[\bar{v}_{1}(|x|)\right]^{\frac{q}{1-q}}-\left[\bar{v}_{1}(t)\right]^{\frac{q}{1-q}}\right) d x\right)^{s \frac{1-q}{q}} \\
& \times d\left(\int_{|x|<t)} v_{2}^{1-p_{2}^{\prime}}\right)^{\frac{s}{p_{2}^{\prime}}} ;
\end{aligned}
$$

(b) $0<q<1=p_{2}, p_{1}>1, \frac{1}{s}=\frac{1}{q}-\frac{1}{p_{1}}-1, r_{1} \geq 1$,

$$
A_{10.1}:=\sup _{t>0}\left(\int_{|x|>t}\left(\int_{|y|>|x|} u\right)^{\frac{r_{1}}{q}}\left(\int_{|y|<|x|)} v_{1}^{1-p_{1}^{\prime}}\right)^{\frac{r_{1}}{q^{\prime}}} v_{1}^{1-p_{1}^{\prime}}(x) d x\right)^{\frac{1}{r_{1}}} \bar{v}_{2}(t),
$$




$$
\begin{aligned}
A_{10.2}:=\sup _{t>0}\left(\int_{|x|>t}\left(\int_{|y|>|x|} u\right)^{\frac{q}{1-q}} u(x)\left(\left[\bar{v}_{2}(|x|)\right]^{\frac{q}{1-q}}-\left[\bar{v}_{2}(t)\right]^{\frac{q}{1-q}}\right) d x\right)^{\frac{1-q}{q}} \\
\times\left(\int_{|x|<t)} v_{1}^{1-p_{1}^{\prime}}\right)^{\frac{1}{p_{1}^{\prime}}} ;
\end{aligned}
$$

$r_{1}<1$

$$
\begin{array}{r}
A_{10.1}:=\left(\int_{[0, \infty]}\left(\int_{|x|>t}\left(\int_{|y|>|x|} u\right)^{\frac{r_{1}}{q}}\left(\int_{|y|<|x|)} v_{1}^{1-p_{1}^{\prime}}\right)^{\frac{r_{1}}{q^{\prime}}} v_{1}^{1-p_{1}^{\prime}}(x) d x\right)^{\frac{s}{r_{1}}} d \bar{v}_{2}^{s}(t)\right)^{\frac{1}{s}}, \\
A_{10.2}^{s}:=\int_{[0, \infty]}\left(\int_{|x|>t}\left(\int_{|y|>|x|} u\right)^{\frac{q}{1-q}} u(x)\left(\left[\bar{v}_{2}(|x|)\right]^{\frac{q}{1-q}}-\left[\bar{v}_{2}(t)\right]^{\frac{q}{1-q}}\right) d x\right)^{s \frac{1-q}{q}} \\
\times d\left(\int_{|x|<t)} v_{1}^{1-p_{1}^{\prime}}\right)^{\frac{s}{p_{1}^{\prime}}} .
\end{array}
$$

ДоКАЗАТЕЛЬСтво. Используя теорему 3 , принцип двойственности и теорему 1 , получим

$$
\begin{aligned}
& \left(\int\left(\int f\right)^{q}\left(\int g\right)^{q} u(x) d x\right)^{\frac{1}{q}} \\
& C=\sup _{g} \sup _{f} \frac{\mathbb{R}^{n} B(|x|)}{\|f\|_{p_{1}, v_{1}}\|g\|_{p_{2}, v_{2}}} \\
& \approx \sup _{g}\|g\|_{p_{2}, v_{2}}^{-1}\left(\int_{\mathbb{R}^{n}} \bar{v}_{1}^{\frac{q}{1-q}}(x)\left(\int_{\mathbb{R}^{n} \backslash B(|x|)}\left(\int_{B(|y|)} g\right)^{q} u(y) d y\right)^{\frac{q}{1-q}}\left(\int_{B(|x|)} g\right)^{q} u(x) d x\right)^{\frac{1-q}{q}} \\
& \approx \sup _{g}\|g\|_{p_{2}, v_{2}}^{-1}\left(\int_{[0, \infty]}\left(\int_{|y|>t}\left(\int_{B(|y|)} g\right)^{q} u(y) d y\right)^{\frac{1}{1-q}} d\left(\bar{v}_{1}^{\frac{q}{1-q}}(t)\right)\right)^{\frac{1-q}{q}} \\
& =\sup _{g}\|g\|_{p_{2}, v_{2}}^{-1}\left[\sup _{h} \frac{\int_{[0, \infty]}\left(\int_{|y|>t}\left(\int_{B(|y|)} g\right)^{q} u(y) d y\right) \mathbf{h}(t) d \bar{v}_{1}^{\frac{q}{1-q}}(t)}{\left(\int_{[0, \infty]} \mathbf{h}^{\frac{1}{q}}(t) d \bar{v}_{1}^{\frac{q}{1-q}}(t)\right)^{q}}\right]^{\frac{1}{q}} \\
& =\sup _{g}\|g\|_{p_{2}, v_{2}}^{-1}\left[\sup _{h} \frac{\int_{\mathbb{R}^{n}}\left(\int_{B(|y|)} g\right)^{q} u(y)\left(\int_{t<|y|} \mathbf{h}(t) d \bar{v}^{\frac{q}{1-q}}(t)\right) d y}{\left(\int_{[0, \infty]} \mathbf{h}^{\frac{1}{q}}(t) d \bar{v}^{\frac{q}{1-q}}(t)\right)^{q}}\right]^{\frac{1}{q}} \\
& =\sup _{h} \frac{\left(\int_{\mathbb{R}^{n}}\left(\int_{\mathbb{R}^{n} \backslash B(|x|)} u(y)\left(\int_{t<|y|} \mathbf{h}(t) d \bar{v}_{1}^{\frac{q}{1-q}}(t)\right) d y\right)^{\frac{r_{2}}{q}}\left(\int_{B(|x|)} v_{2}^{1-p_{2}^{\prime}}\right)^{\frac{r_{2}}{q^{\prime}}} v_{2}^{1-p_{2}^{\prime}}(x) d x\right)^{\frac{1}{r_{2}}}}{\left(\int_{[0, \infty]} \mathbf{h}^{\frac{1}{q}}(t) d \bar{v}_{1}^{\frac{q}{1-q}}(t)\right)^{q}} \\
& \approx \sup _{h}\left[\frac{\left(\int_{\mathbb{R}^{n}}\left(\int_{t<|x|} \mathbf{h}(t) d \bar{v}_{1}^{\frac{q}{1-q}}(t)\right)^{\frac{r_{2}}{q}}\left(\int_{|y|>|x|} u\right)^{\frac{r_{2}}{q}}\left(\int_{B(|x|)} v_{2}^{1-p_{2}^{\prime}}\right)^{\frac{r_{2}}{q^{\prime}}} v_{2}^{1-p_{2}^{\prime}}(x) d x\right)^{\frac{q}{r_{2}}}}{\left(\int_{[0, \infty]} \mathbf{h}^{\frac{1}{q}}(t) d \bar{v}_{1}^{\frac{q}{1-q}}(t)\right)^{q}}\right]^{\frac{1}{q}}
\end{aligned}
$$




$$
\begin{array}{r}
+\sup _{h}\left[\frac{\left(\int_{\mathbb{R}^{n}}\left(\int_{t>|x|} \mathbf{h}(t) d \bar{v}_{1}^{\frac{q}{1-q}}(t)\left(\int_{|y|>t} u\right)\right)^{\frac{r_{2}}{q}}\left(\int_{B(|x|)} v_{2}^{1-p_{2}^{\prime}}\right)^{\frac{r_{2}}{q^{\prime}}} v_{2}^{1-p_{2}^{\prime}}(x) d x\right)^{\frac{q}{r_{2}}}}{\left(\int_{[0, \infty]} \mathbf{h}^{\frac{1}{q}}(t) d \bar{v}_{1}^{\frac{q}{1-q}}(t)\right)^{q}}\right]^{\frac{1}{q}} \\
=: \mathscr{H}_{1}+\mathscr{H}_{2} .
\end{array}
$$

При $\frac{1}{q} \leq \frac{r_{2}}{q} \Leftrightarrow r_{2} \geq 1$ оценим константы $\mathscr{H}_{1}$ и $\mathscr{H}_{2}$. Для константы $\mathscr{H}_{2}$ используем те же рассуждения, что для $H_{1}$ в теореме $12(\mathrm{a})$ :

$$
\begin{array}{r}
\mathscr{H}_{1}=\sup _{t>0}\left(\int_{|x|>t}\left(\int_{|y|>|x|} u\right)^{\frac{r_{2}}{q}}\left(\int_{|y|<|x|)} v_{2}^{1-p_{2}^{\prime}}\right)^{\frac{r_{2}}{q^{\prime}}} v_{2}^{1-p_{2}^{\prime}}(x) d x\right)^{\frac{1}{r_{2}}} \bar{v}_{1}(t), \\
\mathscr{H}_{2}=\sup _{t>0}\left(\int_{|x|>t}\left(\int_{|y|>|x|} u\right)^{\frac{q}{1-q}} u(x)\left(\left[\bar{v}_{1}(|x|)\right]^{\frac{q}{1-q}}-\left[\bar{v}_{1}(t)\right]^{\frac{q}{1-q}}\right) d x\right)^{\frac{1-q}{q}} \\
\times\left(\int_{|x|<t)} v_{2}^{1-p_{2}^{\prime}}\right)^{\frac{1}{p_{2}^{\prime}}} .
\end{array}
$$

При $\frac{r_{2}}{q}<\frac{1}{q} \Leftrightarrow r_{2}<1, \frac{1}{s}=\frac{1}{q}-\frac{1}{p_{2}}-1$ используем теорему 2 из [30], находим

$$
\begin{gathered}
\mathscr{H}_{1}=\left(\int_{[0, \infty]}\left(\int_{|x|>t}\left(\int_{|y|>|x|} u\right)^{\frac{r_{2}}{q}}\left(\int_{|y|<|x|)} v_{2}^{1-p_{2}^{\prime}}\right)^{\frac{r_{2}}{q^{\prime}}} v_{2}^{1-p_{2}^{\prime}}(x) d x\right)^{\frac{s}{r_{2}}} d \bar{v}_{1}^{s}(t)\right)^{\frac{1}{s}}, \\
\mathscr{H}_{2}^{s}=\int_{[0, \infty]}\left(\int_{|x|>t}\left(\int_{|y|>|x|} u\right)^{\frac{q}{1-q}} u(x)\left(\left[\bar{v}_{1}(|x|)\right]^{\frac{q}{1-q}}-\left[\bar{v}_{1}(t)\right]^{\frac{q}{1-q}}\right) d x\right)^{s \frac{1-q}{q}} \\
\times d\left(\int_{|x|<t)} v_{2}^{1-p_{2}^{\prime}}\right)^{\frac{s}{p_{2}^{\prime}}}
\end{gathered}
$$

Теорема 14 (случай $\left.I V_{3}\right)$. Пусть $0<q<1=\min \left(p_{1}, p_{2}\right), \max \left(p_{1}, p_{2}\right)=\infty$. Тогда $C \approx A_{12}$, где

(a) $0<q<1=p_{1}, p_{2}=\infty$

$$
A_{11}:=\left(\int_{[0, \infty]}\left(\int_{|x|>t}\left(\int_{|y|<|x|} v_{2}^{-1}\right)^{q} u(x) d x\right)^{\frac{1}{1-q}} d\left[\bar{v}_{1}(t)\right]^{\frac{q}{1-q}}\right)^{\frac{1-q}{q}}
$$

(b) $0<q<1=p_{2}, p_{1}=\infty$,

$$
A_{11}:=\left(\int_{[0, \infty]}\left(\int_{|x|>t}\left(\int_{|y|<|x|} v_{1}^{-1}\right)^{q} u(x) d x\right)^{\frac{1}{1-q}} d\left[\bar{v}_{2}(t)\right]^{\frac{q}{1-q}}\right)^{\frac{1-q}{q}} .
$$

ДокАЗАТЕльство. Применяя те же рассуждения, что в теореме 13, и используя лемму из $\S 2$ в случаях (ii) и (iii), получим

$$
C=\sup _{g} \sup _{f} \frac{\left(\int_{\mathbb{R}^{n}}\left(\int_{B(|x|)} f\right)^{q}\left(\int_{B(|x|)} g\right)^{q} u(x) d x\right)^{\frac{1}{q}}}{\|f\|_{p_{1}, v_{1}}\|g\|_{p_{2}, v_{2}}}
$$




$$
\begin{gathered}
\approx \sup _{g}\|g\|_{p_{2}, v_{2}}^{-1}\left(\int_{\mathbb{R}^{n}} \bar{v}_{1}^{\frac{q}{1-q}}(x)\left(\int_{\mathbb{R}^{n} \backslash B(|x|)}\left(\int_{B(|y|)} g\right)^{q} u(y) d y\right)^{\frac{q}{1-q}}\left(\int_{B(|x|)} g\right)^{q} u(x) d x\right)^{\frac{1-q}{q}} \\
\approx \sup _{g}\|g\|_{p_{2}, v_{2}}^{-1}\left(\int_{[0, \infty]}\left(\int_{|y|>t}\left(\int_{B(|y|)} g\right)^{q} u(y) d y\right)^{\frac{1}{1-q}} d\left(\bar{v}_{1}^{\frac{q}{1-q}}(t)\right)\right)^{\frac{1-q}{q}} \\
=\sup _{g}\|g\|_{p_{2}, v_{2}}^{-1}\left[\sup _{h} \frac{\int_{[0, \infty]}\left(\int_{|y|>t}\left(\int_{B(|y|)} g\right)^{q} u(y) d y\right) \mathbf{h}(t) d \bar{v}_{1}^{\frac{q}{1-q}}(t)}{\left(\int_{[0, \infty]} \mathbf{h}^{\frac{1}{q}}(t) d \bar{v}^{\frac{q}{1-q}}(t)\right)^{q}}\right]^{\frac{1}{q}} \\
=\sup _{g}\|g\|_{p_{2}, v_{2}}^{-1}\left[\sup _{h} \frac{\int_{\mathbb{R}^{n}}\left(\int_{B(|y|)} g\right)^{q} u(y)\left(\int_{t<|y|} \mathbf{h}(t) d \bar{v}_{1}^{\frac{q}{1-q}}(t)\right) d y}{\left(\int_{[0, \infty]} \mathbf{h}^{\frac{1}{q}}(t) d \bar{v}_{1}^{\frac{q}{1-q}}(t)\right)^{q}}\right. \\
=\left[\int_{\mathbb{R}^{n}}\left(\int_{B(|y|)} v_{2}^{-1}\right)^{q} u(y)\left(\int_{t<|y|} \mathbf{h}(t) d \bar{v}_{1}^{\frac{q}{q}-q}(t)\right) d y\right. \\
\left(\int_{[0, \infty]} \mathbf{h}^{\frac{1}{q}}(t) d \bar{v}_{1}^{\frac{q}{1-q}}(t)\right)^{q} \\
\approx\left(\int_{[0, \infty]}\left(\int_{|x|>t}\left(\int_{|y|<|x|} v_{2}^{-1}\right)^{q} u(x) d x\right)^{\frac{1}{1-q}} d\left[\bar{v}_{1}(t)\right]^{\frac{q}{1-q}}\right)^{\frac{1-q}{q}}
\end{gathered}
$$

Благодарность. Авторы выражают глубокую благодарность рецензенту за ценные замечания.

\section{ЛИТЕРАТУРА}

1. Christ M., Grafakos L. Best constants for two nonconvolution inequalities // Proc. Amer. Math. Soc. 1995. V. 123, N 6. P. 1687-1693.

2. Drábek P., Heinig H. P., Kufner A. Higher dimensional Hardy inequality. Birkhäuser-Verl.: Basel, 2012.

3. Sinnamon G. One-dimensional Hardy-type inequalities in many dimensions // Proc. Roy. Soc. Edinburgh. 1998. V. 128, N 4. Sec. A. P. 833-848.

4. Persson L.-E., Samko S. G. A note on the best constants in some Hardy inequalities // J. Math. Inequal. 2015. V. 9, N 2. P. 437-447.

5. Mamedov F. I., Harman A. On a weighted inequality of Hardy type in spaces $L^{p(\cdot)} / / \mathrm{J}$. Math. Anal. Appl. 2009. V. 353, N 2. P. 521-530.

6. Mamedov F. I., Harman A. On a Hardy type general weighted inequality in spaces $L^{p(\cdot)} / /$ Integral Equations Operator Theory. 2010. V. 66, N 4. P. 565-592.

7. Cruze-Uribe D., Mamedov F. I. On a general weighted Hardy type inequality in the variable exponent Lebesgue spaces // Rev. Mat. Complut. 2012. V. 25, N 2. P. 335-367.

8. Aguilar Cañestro M. I., Ortega Salvador P., Ramírez Torreblanca C. Weighted bilinear Hardy inequalities // J. Math. Anal. Appl. 2012. V. 387, N 1. P. 320-334.

9. Krepela M. Iterating bilinear Hardy inequalities // Proc. Edinb. Math. Soc. 2017. V. 60. P. 955-971.

10. Cwikel M., Kerman R. Positive multilinear operators acting on weighted $L_{p}$ spaces // J. Funct. Anal. 1992. V. 106, N 1. P. 130-144.

11. Grafakos L., Torres R. H. A multilinear Schur test and multiplier operators // J. Funct. Anal. 2001. V. 187, N 1. P. 1-24.

12. Прохоров В. Д., Степанов В. Д., Ушакова Е. П. Интегральные операторы Харди Стеклова // Современные проблемы математики. МИАН. 2016. Т. 22, № 6. С. 1-186. 
13. Джейн П., Канжилал С., Степанов В. Д., Ушакова Е. П. О билинейных операторах Харди - Стеклова // Докл. АН. 2018. Т. 483. С. 602-605.

14. Jain P., Kanjilal S., Stepanov V. D., Ushakova E. P. Bilinear Hardy-Steklov operators // Math. Notes. 2018. V. 104, N 6. P. 823-832.

15. Прохоров Д. В. Об одном классе весовых неравенств, содержащих квазилинейные операторы // Тр. МИАН. 2016. Т. 293. С. 280-295.

16. Степанов В. Д., Шамбилова Г. Э. О билинейных весовых неравенствах с интегральными операторами Вольтерра // Докл. АН. 2019. Т. 486, № 4. С. 416-420.

17. Stepanov V. D., Shambilova G. E. On iterated and bilinear integral Hardy-type operators // Math. Inequal. Appl. 2019. V. 8. P. 47-73.

18. Stepanov V. D., Ushakova E. P. Bilinear Hardy-type inequalities in weighted Lebesgue spaces // Nonlinear Stud. 2018. V. 26, N 4. P. 939-953.

19. Krepela $M$. Bilinear weighted Hardy inequality for nonincreasing functions // Publ. Mat. 2017. V. 61. P. 3-50.

20. Степанов В. Д., Шамбилова Г. Э. О билинейных весовых неравенствах на конусе неубывающих функций // Докл. АН. 2017. Т. 477, № 6. С. 652-656.

21. Степанов В. Д., Шамбилова Г. Э. Редукция билинейных весовых неравенств с операторами интегрирования на конусе неубывающих функций // Сиб. мат. журн. 2018. Т. 59, № 3. C. $639-658$.

22. Bigicli N., Mustafayev R. Ch., Ë Unver T. Multidimensional bilinear Hardy inequalities // Azerbaijan J. Math. 2020. V. 10, N 1. P. 127-161.

23. Jain P., Kanjilal S., Persson L.-E. Hardy-type inequalities over balls in $\mathbb{R}^{N}$ for some bilinear and iterated operators // J. Inequal. Special Functions. 2019. V. 10, N 2. P. 35-48.

24. Goldman M. L., Heinig H. P., Stepanov V. D. On the principle of duality in Lorentz spaces // Canad. J. Math. 1996. V. 48, N 5. P. 959-979.

25. Gogatishvili A., Pick L. Discretization and antidiscretization of rearrangement-invariant norms // Publ. Mat. 2003. V. 47, N 2. P. 311-358.

26. Степанов В. Д., Шамбилова Г. Э. Многомерные билинейные неравенства Харди // Докл. AH. 2019. T. 487, № 5. C. 496-498.

27. Sinnamon G., Stepanov V. D. The weighted Hardy inequality: new proofs and the case $p=1 / /$ J. London Math. Soc. 1996. V. 54, N 2. P. 89-101.

28. Канторович Л. В., Акилов Г. П. Функциональный анализ. М.: Наука, 1984.

29. Гогатишвили А., Степанов В. Д. Редукционные теоремы для весовых интегральных неравенств на конусе монотонных функций // Успехи мат. наук. 2013. Т. 68, № 4. С. 3-68.

30. Прохоров Д. В. Неравенство Харди с тремя мерами // Тр. МИАН. 2006. Т. 255. С. 233-245.

Поступила в редакцию 28 января 2020 г.

После доработки 18 марта 2020 г

Принята к публикации 8 апреля 2020 г.

Степанов Владимир Дмитриевич

Вычислительный центр ДВО РАН,

ул. Ким Ю Чена, 65, Хабаровск 680000

stepanov@mi-ras.ru

Шамбилова Гульдарья Эрмаковна

Московский физико-технический институт

(национальный исследовательский университет),

Институтский пер., 9, г. Долгопрудный 141701 Московской области

shambilova@mail.ru 